\title{
Combined Model-Based Segmentation and Elastic Registration for Accurate Quantification of the Aortic Arch
}

\author{
Andreas Biesdorf ${ }^{1}$, Karl Rohr ${ }^{1}$, Hendrik von Tengg-Kobligk ${ }^{2}$, \\ and Stefan Wörz ${ }^{1}$
}

1 University of Heidelberg, BIOQUANT, IPMB, and DKFZ Heidelberg Dept. Bioinformatics and Functional Genomics, Biomedical Computer Vision Group

${ }^{2}$ German Cancer Research Center (DKFZ) Heidelberg, Dept. of Radiology and University Hospital Heidelberg, Dept. of Diagnostic and Interventional Radiology

\begin{abstract}
Accurate quantification of the morphology of vessels is important for diagnosis and treatment of cardiovascular diseases. We introduce a new approach for the quantification of the aortic arch morphology that combines 3D model-based segmentation with elastic image registration. The performance of the approach has been evaluated using 3D synthetic images and clinically relevant 3D CTA images including pathologies. We also performed a comparison with a previous approach.
\end{abstract}

\section{Introduction}

Diseases of the aortic arch are a major cause of death in the western world and can be diagnosed using, for example, computed tomography angiography (CTA), and treated by minimally-invasive placement using an endovascular graft. For this task, individual morphological parameters such as the centerline position and the vessel diameters have to be quantified. The geometry of the aortic arch can be automatically determined from radiological images by segmentation approaches. A wide spectrum of different approaches exists, for example, approaches based on differential measures, deformable models, or parametric intensity models. Alternatively, segmentation may also be achieved by registration, where an atlas or a model is registered with an image of a patient (e.g., [23]).

In recent years, increased attention has been paid to combined approaches for vessel analysis that integrate both segmentation and registration (e.g., [14518]). Existing approaches can be classified according to the transformation model (e.g., rigid, affine, elastic), the type of information used for registration (e.g., point sets, surfaces, intensities), and whether prior information is employed (e.g., an atlas or a template image). Most approaches use rigid transformation models (e.g., [1]), or splines on a regular grid such as B-Splines (e.g., 4]) and Cardinal-Splines (e.g., 5]). In addition, registration is performed based on, for instance, surfaces (e.g., [5]), point sets (e.g., [8]), centerlines (e.g., [1]), or binarized image volumes (e.g., 4]). Thus, the image intensities are not directly exploited. Moreover, none of the combined approaches uses an incremental tracking scheme or employs a parametric intensity model for vessel segmentation.

T. Jiang et al. (Eds.): MICCAI 2010, Part I, LNCS 6361, pp. 444 451, 2010.

(C) Springer-Verlag Berlin Heidelberg 2010 
In this contribution, we introduce a novel approach for the quantification of the aortic arch morphology from 3D tomographic images. Our approach combines 3D fitting of a parametric intensity model with intensity-based elastic image registration. The image intensities are exploited directly, thus the full intensity information is incorporated. In comparison to a pure model fitting approach (e.g., 6]), the combined approach can cope with a larger spectrum of vessel shapes and even with shapes that deviate significantly from the model used for segmentation. At the same time, our approach is constrained to meaningful shapes by employing a physically-based deformation model for registration. Moreover, our approach can cope with very different shapes of vessel centerlines since an incremental tracking scheme is used. In contrast to atlas- or model-based segmentation (e.g., 23]), our approach does not require a prior segmentation. Instead, the intensity template is automatically generated by model-based segmentation. We have successfully applied our approach to 3D synthetic images and clinically relevant 3D CTA images, and the performance has been evaluated.

\section{Model-Based Segmentation and Elastic Registration}

Our approach for the segmentation of vessels in 3D tomographic images combines model-based segmentation with elastic image registration. The approach is based on an energy-minimizing functional $J_{k}$ corresponding to a vessel segment $k$ :

$$
J_{k}\left(\mathbf{p}_{k}, \mathbf{u}_{k}\right)=J_{M}\left(g_{M}, g_{I, k}^{r o i}, \mathbf{p}_{k}\right)+J_{R}\left(g_{I, k}^{r o i}, g_{M, k}^{r o i}, \mathbf{u}_{k}\right)
$$

The first term $J_{M}$ denotes an intensity similarity measure between a 3D cylindrical intensity model $g_{M}$ with parameters $\mathbf{p}_{k}$ and the intensities $g_{I, k}^{r o i}$ within a region-of-interest (ROI) of a 3D tomographic image $g_{I}$. The second term $J_{R}$ denotes an energy-minimizing functional for elastic registration of $g_{I, k}^{r o i}$ with an image $g_{M, k}^{r o i}$ generated from the 3D intensity model $g_{M}$. The result of elastic registration is described by the deformation field $\mathbf{u}_{k}$. By minimizing the overall functional $J_{k}$, the segmentation result from $3 \mathrm{D}$ model fitting is refined using elastic image registration, while at the same time the registration result is used to improve 3D model fitting. For segmentation of an entire vessel such as the aortic arch, we incrementally minimize $J_{k}$ along the vessel using a tracking approach based on a Kalman filter assuming a linear motion model. Typically, a segmentation result of the aorta consists of several hundreds overlapping vessel segments. Branches can be detected based on a connected components analysis.

The 3D parametric intensity model used in $J_{M}$ represents an ideal sharp 3D cylinder convolved with a 3D Gaussian. The model includes parameters for the width $R$ of the tubular structure and the image blur $\sigma$, and is well-suited to describe the plateau-like intensity structure of thick vessels such as the aorta:

$$
g_{C y l}(\mathbf{x}, R, \sigma)=\Phi\left(\frac{c_{2}-1}{c_{1}}+c_{1}\right), c_{1}=\frac{2}{3} \sigma \frac{\sqrt{\sigma^{2}+r^{2}}}{2 \sigma^{2}+r^{2}}, c_{2}=\sqrt[3]{\frac{R^{2}}{2 \sigma^{2}+r^{2}}}
$$

where $\Phi(x)=\int_{-\infty}^{x}(2 \pi)^{-1 / 2} e^{-\xi^{2} / 2} d \xi$ denotes the Gaussian error function, $\mathbf{x}=$ $(x, y, z)^{T}$, and $r=\sqrt{x^{2}+y^{2}}$. The complete model also incorporates intensity 


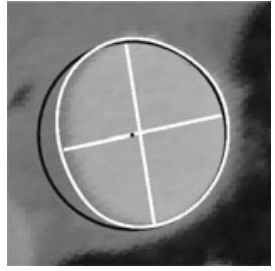

(a)

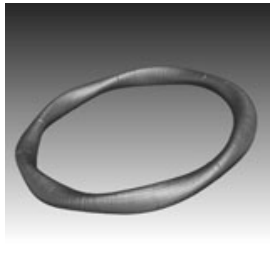

(b)

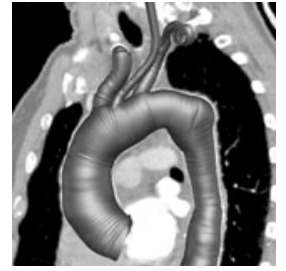

(c)

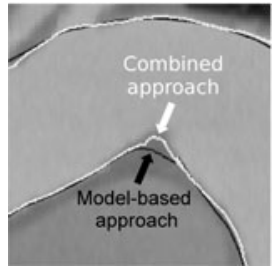

(d)

Fig. 1. (a) Cross-section of a 3D CTA image of an aorta and overlaid result of modelbased segmentation (black) and combined model-based segmentation and elastic registration (white). (b) Segmentation result of the 2D combined approach for a 3D synthetic image of a twisted torus. (c) Segmentation result of the 2D combined approach for a 3D CTA image. (d) Computed vessel contours using the model-based approach (black) and the 2D combined approach (white) for a section of a 3D CTA image.

levels $a_{0}$ (surrounding tissue) and $a_{1}$ (vessel) as well as a $3 \mathrm{D}$ rigid transform $\mathcal{R}$ with rotation $\boldsymbol{\alpha}=(\alpha, \beta, \gamma)^{T}$ and translation $\mathbf{x}_{0}=\left(x_{0}, y_{0}, z_{0}\right)^{T}$, which yields

$$
g_{M}(\mathbf{x}, \mathbf{p})=a_{0}+\left(a_{1}-a_{0}\right) g_{C y l}\left(\mathcal{R}\left(\mathbf{x}, \boldsymbol{\alpha}, \mathbf{x}_{0}\right), R, \sigma\right)
$$

with 10 parameters $\mathbf{p}=\left(R, a_{0}, a_{1}, \sigma, \alpha, \beta, \gamma, x_{0}, y_{0}, z_{0}\right)^{T}[6]$.

The cylindrical model $g_{M}$ can accurately represent a vessel segment if the vessel has circular cross-sections. However, the model may be inaccurate in the case of non-circular cross-sections (e.g., Fig. 19, black contour). To improve the accuracy between the model and the true vessel shape in this case, we suggest using elastic registration of an image $g_{M, k}^{r o i}$ generated from the $3 \mathrm{D}$ intensity model $g_{M}$ with a ROI of the original image $g_{I, k}^{r o i}$. The result of elastic registration is a deformation field $\mathbf{u}_{k}$ which can be used to compute a refined vessel contour and centerline position (e.g., Fig. 19, white contour). To limit the final segmentation result in (11) to physically meaningful shapes, the deformations $\mathbf{u}_{k}$ are computed based on Gaussian elastic body splines (e.g., [7]).

\subsection{Optimization of the Energy-Minimizing Functional $\boldsymbol{J}_{\boldsymbol{k}}$}

The functional in (1) is optimized by an iterative scheme which alternatingly minimizes $J_{M}$ and $J_{R}$ for each vessel segment $k$ to obtain estimates for the model parameters $\mathbf{p}_{k}$ and the deformation field $\mathbf{u}_{k}$ (see Fig. 2). For a vessel segment $k$, we estimate $\mathbf{p}_{k}$ by least-squares model fitting of $g_{M}$ to the image intensities $g_{I, k}^{r o i}$ by minimizing

$$
J_{M}\left(\mathbf{p}_{k}\right)=\sum_{\mathbf{x} \in g_{I, k}^{r o i}}\left(g_{M}\left(\mathbf{x}, \mathbf{p}_{k}\right)-g_{I, k}^{r o i}(\mathbf{x})\right)^{2}
$$

using the method of Levenberg-Marquardt. To compute the deformation field $\mathbf{u}_{k}$, we generate an image $g_{M, k}^{r o i}$ from the fitted intensity model $g_{M}$ and perform intensity-based registration with $g_{I, k}^{r o i}$ by minimizing 


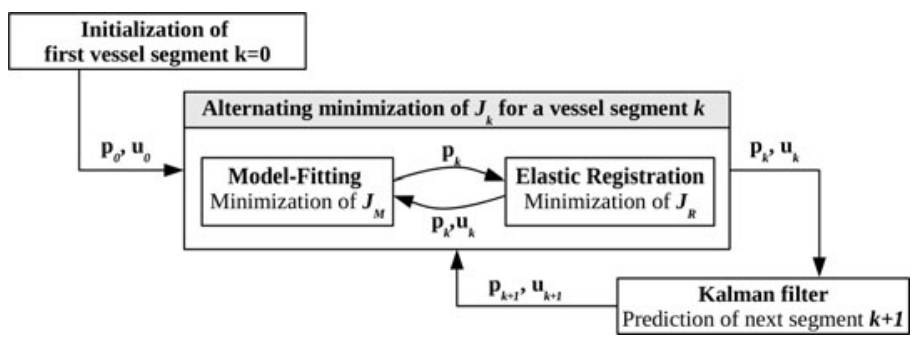

Fig. 2. Incremental combined vessel segmentation approach

$$
J_{R}\left(\mathbf{u}_{k}\right)=J_{\text {Data }, I}\left(g_{I, k}^{r o i}, g_{M, k}^{r o i}, \mathbf{u}_{k}^{I}\right)+\lambda_{I} J_{I}\left(\mathbf{u}_{k}, \mathbf{u}_{k}^{I}\right)+\lambda_{E} J_{\text {Elastic }}\left(\mathbf{u}_{k}\right)
$$

where $\lambda_{I}$ and $\lambda_{E}$ are scalar weights. We use $\lambda_{E}=0.1$ while $\lambda_{I}$ is estimated automatically. The first term $J_{\text {Data,I }}$ describes the intensity-based similarity measure between $g_{I, k}^{r o i}$ and $g_{M, k}^{r o i}$. Since $g_{M}$ incorporates intensity levels $a_{0}$ and $a_{1}$, minimization of $J_{\text {Data,I }}$ can be considered a monomodal registration problem. Therefore, we use the sum-of-squared intensity differences for $J_{\text {Data,I }}$ (cf. (4)). With the second term $J_{I}$, the intensity-based deformation field $\mathbf{u}_{k}^{I}$ is coupled with the final deformation field $\mathbf{u}_{k}$ using a weighted Euclidean distance. The third term $J_{\text {Elastic }}$ represents the regularization of the deformation field according to the Navier equation of linear elasticity. Optimization of $J_{R}$ is performed alternatingly w.r.t. $\mathbf{u}_{k}^{I}$ and $\mathbf{u}_{k}$. Note that the functional (5) has been formulated such that for the minimization of $\lambda_{I} J_{I}+\lambda_{E} J_{\text {Elastic }}$ w.r.t. $\mathbf{u}_{k}$ an analytic solution can be derived [7. For the minimization w.r.t. $\mathbf{u}_{k}^{I}, J_{\text {Data,I}}+J_{I}$ has to be minimized for which we use the method of Levenberg-Marquardt.

The result of elastic registration is used to improve the result of model fitting by re-estimating the model parameters $\mathbf{p}_{k}$ including the radius $R$, the orientation $\boldsymbol{\alpha}$, as well as the translation $\mathbf{x}_{0}$. To update the radius $R$, we compute the mean radius along the vessel contour based on $\mathbf{u}_{k}$. For the translation $\mathbf{x}_{0}$ we obtain new estimates based on the deformation $\mathbf{u}_{k}\left(\mathbf{x}_{0}\right)$. The orientation $\boldsymbol{\alpha}$ can be reestimated using finite differences of the positions of two points close to $\mathbf{x}_{0}$.

Based on the updated parameter vector $\mathbf{p}_{k}$ and the deformation field $\mathbf{u}_{k}$, we again perform model-based segmentation with subsequent elastic registration for minimizing $J$. Note that now, model fitting is performed on a deformed image which is obtained by applying $\mathbf{u}_{k}$ to $g_{I, k}^{r o i}$. Performing model fitting on the deformed image has the advantage that the model parameters can be estimated more accurately since the deformed image is more similar to the underlying model than the original image. This alternating optimization is repeated until the results of model fitting and elastic registration converge for a vessel segment $k$. After convergence and having estimated the parameters for the current vessel segment, a new parameter vector $\mathbf{p}_{k+1}$ is predicted based on a Kalman filter and used as initialization for the next vessel segment along a vessel. For initialization of $\mathbf{u}_{k+1}$ in the next iteration, the current deformation field $\mathbf{u}_{k}$ is used. 


\subsection{Exploiting 2D and 3D Image Information}

With our combined approach for vessel segmentation there are different possibilities to exploit the intensity information. We have developed two approaches, a $3 \mathrm{D}$ and a 2D approach, which differ w.r.t. computational efficiency and segmentation accuracy. The first approach performs model fitting within a 3D ROI and uses 3D image registration of the 3D ROI. Since with this approach all image information within a 3D ROI is exploited, the accuracy as well as the computational complexity is expected to be relatively high. The second variant uses 3D model fitting only for estimating the initial 3D orientation $\boldsymbol{\alpha}$, while $J_{k}$ in (11) is minimized based on model fitting and image registration of $2 \mathrm{D}$ image cross-sections orthogonal to the vessel centerline. Compared to the 3D approach, the computational complexity of the $2 \mathrm{D}$ approach is significantly lower.

\section{Experimental Evaluation}

We have applied our approach to 240 3D synthetic images and 17 clinically relevant 3D CTA images of the human thorax. To quantify the segmentation accuracy, we have computed the mean errors for clinically relevant measures comprising the minimum, mean, and maximum vessel diameters, $\bar{e}_{D, \text { min }}, \bar{e}_{D, \text { mean }}$, and $\bar{e}_{D, \max }$, respectively, as well as the mean error for the centerline position $\bar{e}_{\mathbf{x}_{0}}$. Note that, in general, vessel cross-sections are noncircular. To compute $\bar{e}_{D, \min }$, $\bar{e}_{D, \text { mean }}$, and $\bar{e}_{D, \max }$, we have defined a diameter by the length of a straight line that connects two points on the vessel boundary and that passes through the center $\mathbf{x}_{0}$. The error measures for the diameters were determined by evaluating a sample of 1000 different diameters of a cross-section where the directions of the diameters are equiangularly distributed. $e_{\mathbf{x}_{0}}$ is defined as Euclidean distance between the true center of the vessel and the estimated position $\mathbf{x}_{0}$. For all measures, we have computed mean errors by averaging over all vessel segments.

\subsection{D Synthetic Images}

In a first experiment, we have generated two different sets of 3D synthetic images. The first set contains 120 different images of straight twisted cylinders with elliptical cross-sections that differ in radii and the level of Gaussian image noise $\left(\sigma_{n}=0,1,3,5,20\right)$, and have a size of $200 \times 200 \times 200$ voxels. The second image set contains 120 different images of twisted tori with elliptical cross-sections that also differ in radii and the level of Gaussian image noise, and have a size of $400 \times 400 \times 200$ voxels (see Fig. 1b for an example). Table 1 shows the results of the segmentation accuracy of the two new combined approaches (2D and $3 \mathrm{D})$ in comparison to a previous model-based approach [6].

Segmentation results: 120 twisted cylinders. For $\bar{e}_{\mathbf{x}_{0}}$ we obtain similar very good results for the new approaches and the previous approach with subvoxel accuracy of $\bar{e}_{\mathbf{x}_{0}} \leq 0.02$ voxels. For $\bar{e}_{D \text {,mean }}$, the results of the approaches are comparable, while the $2 \mathrm{D}$ combined approach yields the best result. For 
$\bar{e}_{D, \min }$ and $\bar{e}_{D, \max }$, the accuracy of the new approaches is significantly better than that of the previous approach. In comparison to the previous approach, the $2 \mathrm{D}$ and $3 \mathrm{D}$ combined approaches yield improvements of $61 \%-66 \%$ for $\bar{e}_{D, \text { min }}$ and $\bar{e}_{D, \max }$.

Segmentation results: 120 twisted tori. For $\bar{e}_{D, \min }, \bar{e}_{D, \text { mean }}$, and $\bar{e}_{D, \max }$, the previous approach yields similar results as for the first image set with $\bar{e}_{D, \text { min }}=3.14$ voxels, $\bar{e}_{D, \text { mean }}=0.09$ voxels, and $\bar{e}_{D, \max }=3.83$ voxels. However, for the $2 \mathrm{D}$ combined approach we obtain a significant improvement for all diameter measures with $\bar{e}_{D, \text { min }}=0.61$ voxels, $\bar{e}_{D, \text { mean }}=0.05$ voxels, and $\bar{e}_{D, \max }=0.88$ voxels, which is an improvement of $77 \%-80 \%$ for $\bar{e}_{D, \min }$ and $\bar{e}_{D, \max }$ as well as $44 \%$ for $\bar{e}_{D, \text { mean }}$ compared to the previous approach. The 3D combined approach, however, yields the best result for all diameter measures with $\bar{e}_{D, \text { min }}=0.37$ voxels, $\bar{e}_{D, \text { mean }}=0.04$ voxels, and $\bar{e}_{D, \max }=0.52$ voxels, which, in comparison to the previous approach, are improvements of $88 \%, 56 \%$, and $86 \%$, respectively. For $\bar{e}_{\mathbf{x}_{0}}$, all approaches yield very good results with subvoxel accuracy, however the $3 \mathrm{D}$ combined approach yields the best result with $\bar{e}_{\mathbf{x}_{0}}=0.08$ voxels, which is an improvement of $50 \%$ compared to the previous approach and $38 \%$ compared to the $2 \mathrm{D}$ combined approach.

\subsection{D CTA Images}

In a second experiment, we applied our approach to two different sets of 3D CTA images of the thorax. The first set of images contains ten 3D CTA images of patients with only slight pathologies. The second set of images contains seven 3D CTA images of patients with severe pathologies such as aneurysms or highly curved vessel centerlines. The CTA images comprise 619 to 829 slices with a size of $512 \times 512$ voxels. For evaluation, manual segmentation by a radiologist was performed for 15 of the 3D CTA images, while two of the images were segmented by a trained observer. Table 2 shows the results for the two new combined approaches and a previous model-based approach [6] for both sets of images.

Segmentation results: Ten 3D CTA images. For the first set of 3D CTA images and for $\bar{e}_{D, \min }$ as well as $\bar{e}_{D, \max }$ the accuracy of our new approaches is significantly better than that of the previous approach, while the best result is obtained for the $3 \mathrm{D}$ combined approach. For $\bar{e}_{D, \text { mean }}$ and $\bar{e}_{\mathbf{x}_{0}}$ we obtain similar good results for the new approaches and the previous approach while for $\bar{e}_{D, \text { mean }}$ the best result is obtained for the $3 \mathrm{D}$ combined approach and for $\bar{e}_{\mathbf{x}_{0}}$

Table 1. Mean errors for the diameters $\bar{e}_{D, \text { min }}, \bar{e}_{D, \text { mean }}$, and $\bar{e}_{D, \max }$, and the centerline position $\bar{e}_{\mathbf{x}_{0}}$ for different approaches

\begin{tabular}{|l||c|c|c|c||c|c|c|c|}
\hline \multicolumn{1}{|c||}{ Accuracy } & \multicolumn{3}{c||}{$\mathbf{1 2 0}$ twisted cylinders } & \multicolumn{4}{c|}{120 twisted tori } \\
\cline { 2 - 10 } Approach & $\bar{e}_{D, \text { min }}$ & $\bar{e}_{D, \text { mean }}$ & $\bar{e}_{D, \text { max }}$ & $\bar{e}_{\mathbf{x}_{0}}$ & $\bar{e}_{D, \text { min }}$ & $\bar{e}_{D, \text { mean }}$ & $\bar{e}_{D, \text { max }}$ & $\bar{e}_{\mathbf{x}_{0}}$ \\
\hline \hline Model-based approach & 3.01 & 0.09 & 3.67 & 0.01 & 3.14 & 0.09 & 3.83 & 0.16 \\
\hline 2D combined approach & 1.04 & 0.07 & 1.42 & 0.02 & 0.61 & 0.05 & 0.88 & 0.13 \\
\hline 3D combined approach & 1.02 & 0.09 & 1.42 & 0.01 & 0.37 & 0.04 & 0.52 & 0.08 \\
\hline
\end{tabular}




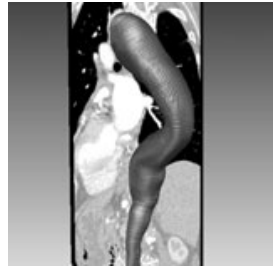

(a)

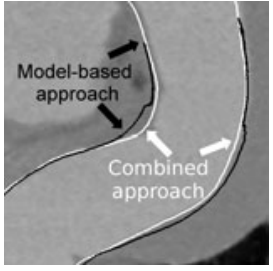

(b)

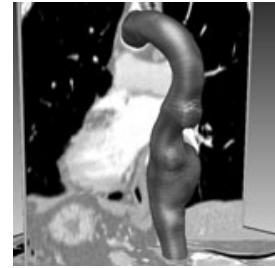

(c)

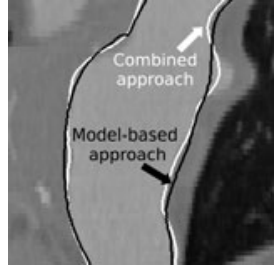

(d)

Fig. 3. (a), (c) Segmentation results of the 3D combined approach for two 3D CTA images showing a pathology. (b), (d) Vessel contours using the model-based approach (black) and the 3D combined approach (white) for a section of the 3D CTA images.

the best result is obtained for the $2 \mathrm{D}$ combined approach. In Fig. 11, d we show the segmentation result of the $2 \mathrm{D}$ combined approach for a $3 \mathrm{D}$ CTA image as well as a section of the same image and the result of the $2 \mathrm{D}$ combined approach in comparison to a previous approach. It can be seen that the new approach yields a significant improvement in highly curved regions.

Segmentation results: Seven 3D CTA images with pathologies. For the second set of 3D CTA images, we consistently obtain more accurate results for the new combined approaches. For $\bar{e}_{D, \text { min }}, \bar{e}_{D, \text { mean }}$, and $\bar{e}_{D, \text { max }}$ the accuracy of the new approaches is significantly better than that of the previous approach, while the best result is obtained for the 3D combined approach with improvements of $21 \%$ to $57 \%$ compared to the previous approach as well as improvements of $9 \%$ to $15 \%$ compared to the $2 \mathrm{D}$ combined approach. For $\bar{e}_{\mathbf{x}_{0}}$, the $2 \mathrm{D}$ combined approach the $2 \mathrm{D}$ combined approach consistently yields the most accurate result. In Fig. 3 we show segmentation results of the 3D combined approach for two 3D CTA images as well as sections of the same images and the result of the new approach in comparison to the previous approach. It can be seen that the new approach yields a significant improvement. Note that for the image in Fig. 3b, only the new approach succeeds to fully segment the pathology, while the previous approach fails to segment the last part of the pathology.

Overall, it turns out that for $\bar{e}_{D, \text { min }}$ and $\bar{e}_{D, \max }$ the new 2D and $3 \mathrm{D}$ combined approaches yield more accurate results than the previous approach. For $\bar{e}_{\mathbf{x}_{0}}$, the $2 \mathrm{D}$ combined approach yields the best result, while for $\bar{e}_{D, \text { mean }}$, the $3 \mathrm{D}$ combined approach yields the best result. The computation time for a vessel segment on a

Table 2. Mean errors for the diameters $\bar{e}_{D, \text { min }}, \bar{e}_{D, \text { mean }}$, and $\bar{e}_{D, \max }$, and the centerline position $\bar{e}_{\mathbf{x}_{0}}$ for different approaches

\begin{tabular}{|l||c|c|c|c||c|c|c|c|}
\hline \multicolumn{1}{|c||}{ Accuracy } & \multicolumn{3}{c||}{ Ten 3D CTA images } & \multicolumn{3}{c|}{ Seven pathologies } \\
\cline { 2 - 10 } Approach & $\bar{e}_{D, \text { min }}$ & $\bar{e}_{D, \text { mean }}$ & $\bar{e}_{D, \text { max }}$ & $\bar{e}_{\mathbf{x}_{0}}$ & $\bar{e}_{D, \text { min }}$ & $\bar{e}_{D, \text { mean }}$ & $\bar{e}_{D, \text { max }}$ & $\bar{e}_{\mathbf{x}_{0}}$ \\
\hline \hline Model-based approach & 5.40 & 2.27 & 1.67 & 0.65 & 7.42 & 2.32 & 6.15 & 1.00 \\
\hline 2D combined approach & 4.67 & 2.38 & 1.58 & 0.47 & 5.19 & 1.89 & 3.14 & 0.63 \\
\hline 3D combined approach & 4.24 & 2.19 & 1.46 & 0.83 & 4.70 & 1.83 & 2.66 & 0.96 \\
\hline
\end{tabular}


2.40 GHz Intel Core 2 Quad CPU is about 1-2 seconds for the 2D approach and about 50-60 seconds for the 3D approach.

\section{Discussion}

We have introduced a new approach for the quantification of the aortic arch from 3D CTA images that combines fitting of a parametric intensity model with intensity-based elastic image registration. We have demonstrated the applicability of our approach using 3D synthetic images and clinically relevant 3D CTA images. From the experiments it turned out that the new combined approach consistently yields more accurate segmentation results than a previous segmentation approach for the minimum and maximum diameters, which are the most relevant clinical measures. For real 3D CTA images, the 2D combined approach is most accurate for estimating the centerline position, while the $3 \mathrm{D}$ combined approach is most accurate for estimating the diameters. It also turned out that for the new approach significant improvements are obtained for difficult segmentation tasks, in particular, for pathologies and highly curved vessel centerlines.

Acknowledgment. Support of the Deutsche Forschungsgemeinschaft (DFG) within the project QuantVessel (RO 2471/6) is gratefully acknowledged.

\section{References}

1. Aylward, S.R., Jomier, J., Weeks, S., Bullitt, E.: Registration and Analysis of Vascular Images. Internat. Journal of Computer Vision 55(2/3), 123-138 (2003)

2. Barber, D., Oubel, E., Frangi, A., Hose, D.: Efficient computational fluid dynamics mesh generation by image registration. Med. Imag. Anal. 11(5), 648-662 (2007)

3. Isgum, I., Staring, M., Rutten, A., Prokop, M., Viergever, M., van Ginneken, B.: Multi-Atlas-Based Segmentation With Local Decision Fusion - Application to Cardiac and Aortic Segmentation in CT Scans. IEEE Trans. Med. Imaging 28(7), 1000$1010(2009)$

4. Kang, D.G., Suh, D., Ra, J.: Three-Dimensional Blood Vessel Quantification via Centerline Deformation. IEEE Trans. Med. Imaging 28(3), 405-414 (2009)

5. Wong, W., Chung, A.: Augmented Vessels for Quantitative Analysis of Vascular Abnormalities and Endovascular Treatment Planning. IEEE Trans. Med. Imaging 25(6), 665-684 (2006)

6. Wörz, S., Rohr, K.: Segmentation and Quantification of Human Vessels Using a 3-D Cylindrical Intensity Model. IEEE Trans. on Image Processing 16(8), 1994-2004 (2007)

7. Wörz, S., Rohr, K.: Hybrid Physics-Based Elastic Image Registration Using Approximating Splines. In: Reinhardt, J., Pluim, J. (eds.) Proc SPIE Medical Imaging 2008: Image Processing, San Diego, CA/USA, pp. 6914-6930 (February 2008)

8. Zhao, F., Zhang, H., Wahle, A., Matthew, T.T., Stolpen, A.H., Scholz, T.D., Sonka, M.: Congenital aortic disease: 4D magnetic resonance segmentation and quantitative analysis. Medical Image Analysis 13(3), 483-493 (2009) 